\title{
Nonspecific Interstitial Pneumonia
}

National Cancer Institute

\section{Source}

National Cancer Institute. Nonspecific Interstitial Pneumonia. NCI Thesaurus. Code C35717.

Idiopathic interstitial pneumonia characterized by chronic inflammation and fibrosis in the interstitial lung tissue. It includes cases that cannot be classified into one of the other types of idiopathic interstitial pneumonia. 